\title{
ECUACIONES PREDICTORAS DE LA COMPOSICIÓNTISULAR DE LA CANAL EN DOS RAZAS OVINAS CANARIAS
}

\author{
PREDICTING EQUATIONS FOR TISULAR COMPOSITION IN CARCASS OF TWO \\ CANARY SHEEP BREEDS
}

\begin{abstract}
Camacho, A $^{1}$., Pérez, V. ${ }^{1}$, Mata, J. ${ }^{1}$ y Bermejo, L.A. ${ }^{1}$
${ }^{1}$ Escuela Técnica Superior de Ingeniería Agraria. Universidad de La Laguna. Tenerife. España. acamacho@ull.es
\end{abstract}

Palabras CLAVE Adicionales

Razas locales. Despiece.

\section{RESUMEN}

Se estudiaron dos razas ovinas locales: la Canaria y la Canaria de Pelo. Los animales se criaron en sistema intensivo. Se consideraron dos pesos vivos de sacrificio $(15,30 \pm 0,67$ y $24,23 \pm$ $0,69 \mathrm{~kg}$ ) y dos sexos. Se determinaron las correlaciones entre los porcentajes de los tejidos de la canal y los de las piezas obtenidas de la media canal izquierda. Se obtuvieron ecuaciones para predecir la composición tisular de la canal, a partir de las piezas con mejores coeficientes de regresión. El costillar fue la pieza que mejor predijo el músculo y la grasa. La pierna fue mejor predictora para el hueso.

\section{SUMMARY}

Two local sheep breeds were studied: the Canarian and the Canarian Haired. Animals were intensively reared. Two slaughter weights were considered ( 15 and $24 \mathrm{~kg}$ live weight). Correlations among carcass tissue percentage and tissue percentage in the seven joints in the left halfcarcass were obtained. Equations were obtained to predict carcass tissue composition using most correlated joints. Ribs was the best predictor joint of muscle and fat. Leg was the best predictor of bone.

\section{INTRODUCCIÓN}

La presencia del ganado ovino en las Islas Canarias es muy antigua (Delgado et al., 1998). Según el Catálogo Oficial de Ra-
AdDitional KEYWORDS

Local breeds. Jointing.

zas de España (R.D. 1682/1997), existen tres razas ovinas autóctonas de protección especial. Éstas son la raza Canaria (aptitud leche-carne) y la raza Canaria de Pelo (aptitud cárnica), que son las que cuentan con más efectivos y tienen presencia en varias islas, y la raza Palmera, localizada sólo en la isla de la Palma. El censo (ISTAC, 2006) muestra un aumento en esta especie del $61 \%$ en los últimos trece años, hecho que la sitúa como la segunda especie doméstica en importancia numérica, con 110945 cabezas. Este incremento se debe al interés que ha despertando en los últimos años la raza ovina de pelo (Camacho et al., 2007), cuya explotación asociada al cultivo de la platanera es frecuente (Pérez, 1992). Canarias carece de tradición en la producción de carne ovina. Las razas existentes pueden ser la base de productos cárnicos de calidad diferenciados, tal y como sucede en otras regiones españolas, como Aragón, CastillaLeón y Castilla-La Mancha, entre otras. El Instituto Canario de Investigaciones Agrarias, y las dos universidades canarias, están estudiando la calidad cárnica de la raza Canaria y la raza Canaria de Pelo.

Por lo costoso y laborioso que resulta conocer la composición tisular de la canal, a través de la disección completa de la media 
Tabla I. Medias ( \pm desviación estándar) de la composición tisular de la media canal izquierda (MCI) de la raza Canaria y raza Canaria de Pelo. (Means ( \pm standard deviation) tissue composition of left half-carcass in Canarian breed and Canarian Haired breed).

\begin{tabular}{|c|c|c|c|c|c|}
\hline \multirow[t]{2}{*}{ Tejidos (\%) } & \multicolumn{2}{|c|}{ Raza Canaria } & \multicolumn{2}{|c|}{ Raza Canaria de Pelo } & \multirow[t]{2}{*}{ Sig. } \\
\hline & $15 \mathrm{~kg}(\mathrm{n}=21)$ & $24 \mathrm{~kg}(\mathrm{n}=20)$ & $15 \mathrm{~kg}(\mathrm{n}=20)$ & $24 \mathrm{~kg}(\mathrm{n}=19)$ & \\
\hline Músculo & $48,96 \pm 4,28$ & $49,03 \pm 3,36$ & $53,85 \pm 3,72$ & $55,34 \pm 3,15$ & ns \\
\hline Grasa total & $24,67 \pm 4,11$ & $25,58 \pm 2,96$ & $17,61 \pm 2,63$ & $19,16 \pm 2,41$ & ns \\
\hline Hueso & $21,70 \pm 1,93$ & $20,27 \pm 1,24$ & $22,55 \pm 1,33$ & $20,29 \pm 1,30$ & $\mathrm{~ns}$ \\
\hline
\end{tabular}

ns: no significativo.

canal izquierda, algunos investigadores han optado por estimarla a partir de la disección de la espalda, dados los elevados coeficientes de correlación entre su composición y la de la canal (Boccard et al., 1976; Diestre, 1985; Sañudo, 1980); aunque la valoración de la composición de la canal a través de esta pieza maximiza el músculo y minimiza la grasa (Sañudo, 1980; López, 1987; Sañudo et al., 1989). También se ha intentado predecir a partir de ciertas medidas objetivas de conformación de la canal y el engrasamiento (Luaces et al, 2008; Díaz, 2001). No obstante, Kempster (1981) señala que la predicción a partir de una pieza es más exacta que la obtenida a partir de medidas. Diferentes autores (Ruiz de Huidobro y Cañeque, 1994; Luaces et al, 2008; Miguélez et al., 2006; Díaz, 2001) han abordado trabajos para conocer la composición tisular de la canal a partir de ecuaciones de predicción, obtenidas de las piezas de la canal cuya composición tisular está altamente correlacionada con la de la canal. Se estima conveniente obtener ecuaciones de predicción en nuestros genotipos autóctonos, para evitar los errores que conlleva la aplicación de otras ecuaciones, calculadas para otros genotipos y rangos de peso (Luaces et al., 2008). Este trabajo pretende determinar las ecuaciones que pueden tener una buena predicción de la composición tisular de la canal, en las dos razas estudiadas.

\section{MATERIAL Y MÉTODOS}

Se utilizaron 80 corderos: 41 de la raza Canaria (21 machos y 20 hembras) y 39 de la raza Canaria de Pelo (20 machos y 19 hembras). Los corderos se sacrificaron con un

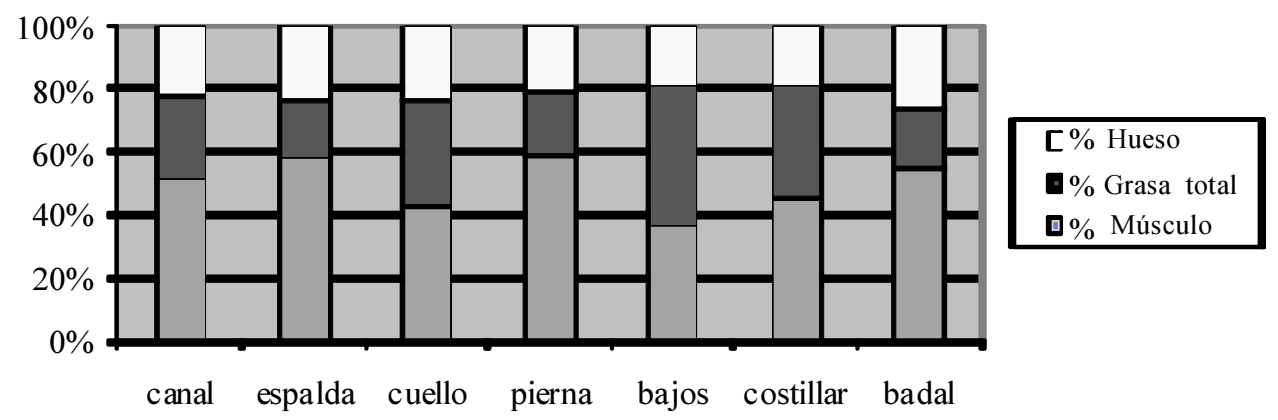

Figura 1. Composición tisular de la media canal izquierda y sus piezas de la raza Canaria. (Tisular composition of half carcass and joints in Canarian breed). 


\section{PREDICCIÓN DE LA COMPOSICIÓN TISULAR DE LA CANAL EN OVINOS CANARIOS}

peso medio de $15,30 \pm 0,67$ ( 21 machos y 20 hembras) y $24,23 \pm 0,69 \mathrm{~kg}$ (20 machos y 20 hembras) de peso vivo. Los corderos nacieron y se criaron en la explotación ovina de tipo intensivo, ubicada al sur de la isla de Tenerife $(2807 \mathrm{Ny} 1635 \mathrm{~W})$, concretamente en las Galletas (Arona), a unos $200 \mathrm{~m}$ sobre el nivel del mar. La temperatura media anual en la zona fue de $20,2^{\circ} \mathrm{C}$ y una humedad relativa del $72 \%$. Los corderos fueron pesados e identificados con un collar numerado dentro de las $24 \mathrm{~h}$ siguientes al nacimiento. A la semana de vida recibieron un choque vitamínico de $\mathrm{A}, \mathrm{D}_{3}, \mathrm{y}$ E, y al mes fueron desparasitados y vacunados de enterotoxemia. Los corderos se mantuvieron en las condiciones de producción habituales de la zona, comenzaron a recibir pienso de iniciación ( 0,9 UF, $18 \%$ PB) y heno a partir de los 15 días de vida. A los 40 días se pasó a un pienso de cebo ( $0,9 \mathrm{UF}, 16 \% \mathrm{~PB})$. Los animales permanecieron con las madres hasta los $16 \mathrm{~kg}$, momento en que fueron separados. Cuando los animales alcanzaban el peso de sacrificio eran trasladados al matadero insular de Tenerife, ubicado a unos $100 \mathrm{~km}$ de la granja. Tras $12 \mathrm{~h}$ de ayuno, se procedió al sacrificio siguiendo el protocolo oficial de sacrificio y faenado descrito por ColomerRocher et al. (1988). Se obtuvo una canal con presencia de riñones, grasa pélvicorrenal, sin cabeza ni autópodos. Tras $24 \mathrm{~h}$ de oreo en cámara refrigerada a $4^{\circ} \mathrm{C}$, la canal se dividió en dos mitades, y se procedió al despiece de la media canal izquierda (MCI), siguiendo el método de Colomer-Rocher $e t$ al. (1988), resultando siete regiones anatómicas diferentes: espalda, bajos, pierna, cuello, costillar, badal y cola. Estas piezas se envasaron en bolsas plásticas individuales, y se congelaron $\left(-18^{\circ} \mathrm{C}\right)$ hasta el momento de su disección, en que se diferenciaron los siguientes tejidos: músculo $(\mathrm{M})$, hueso (H), grasa subcutánea (GS), grasa intermuscular (GI) y despojos (D). La suma de las grasas subcutánea e intermuscular constituyeron la grasa total (GT).

Para el análisis de los datos se usó el programa estadístico SPSS 15.0 (SPSS, 2006). Para cada tipo de cordero se estudiaron las correlaciones entre los tejidos de la media canal izquierda y las diferentes piezas. Se realizó un análisis de regresión lineal múltiple con paso adelante, para determinar las ecuaciones predictivas de la composición tisular de la canal (variable dependiente) a partir de los componentes tisulares (variables independientes) de la pieza diseccionada, para las cuatro tipos de canales (interacción raza*peso) y para los sexos (interacción raza*sexo).

\section{RESULTADOSYDISCUSIÓN}

En la tabla I se presentan los resultados de la composición tisular para los cuatro

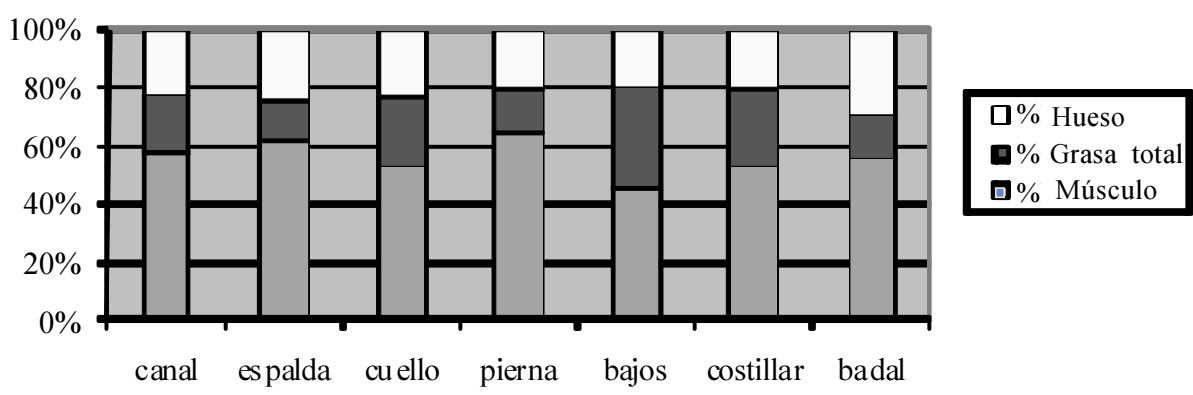

Figura 2. Composicion tisular de la media canal izquierda y sus diferentes piezas de la raza Canaria de Pelo. (Tisular composition of half carcass and joints in Canarian breed). 


\section{CAMACHO, PÉREZ, MATA YBERMEJO}

tipos de canales estudiadas (interacción raza* peso). En ambas razas, al aumentar el peso de sacrificio aumenta el porcentaje de músculo y grasa, y desciende el porcentaje de hueso. La cantidad de grasa está estrechamente relacionada con el peso vivo (Blázquez et al., 2001; Díaz, 2001) aumentando su cantidad a medida que aumenta el

Tabla II. Correlaciones entre los componentes titulares (\%) de las diferentes piezas y los de la canal en la raza Canaria. (Correlations among tissue composition of different joints and tissue composition of carcass in Canarian breed).

\begin{tabular}{|c|c|c|c|c|c|c|}
\hline \multirow{2}{*}{$\begin{array}{l}\text { Pieza } \\
\text { Tejidos }\end{array}$} & \multicolumn{3}{|c|}{$15 \mathrm{~kg}$} & \multicolumn{3}{|c|}{$24 \mathrm{~kg}$} \\
\hline & Músculo & Grasa total & Hueso & Músculo & Grasa total & Hueso \\
\hline \multicolumn{7}{|l|}{ Costillar } \\
\hline Músculo & $0,945^{\star * *}$ & $-0,922^{* * *}$ & 0,015 & $0,907^{\star \star \star}$ & $-0,842^{* * *}$ & $0,503^{*}$ \\
\hline Grasa total & $-0,891^{* * *}$ & $0,884^{* * *}$ & $-0,114$ & $-0,821^{* * *}$ & $0,915^{\star *}$ & $-0,457^{*}$ \\
\hline Grasa intermuscular & $-0,708^{* * *}$ & $0,744^{* * *}$ & $-0,126$ & $-0,417$ & $0,564^{* * *}$ & $-0,099$ \\
\hline Grasa subcutánea & 0,094 & $-0,166$ & 0,074 & $-0,359$ & 0,301 & $-0,331$ \\
\hline Hueso & $-0,163$ & 0,130 & $0,551^{*}$ & 0,156 & $-0,294$ & $0,439^{*}$ \\
\hline \multicolumn{7}{|l|}{ Espalda } \\
\hline Músculo & $0,531^{*}$ & $-0,487^{\star}$ & $-0,060$ & $0,671^{* * *}$ & $-0,674^{* * *}$ & 0,099 \\
\hline Grasa total & $-0,593^{\star *}$ & $0,630^{\star *}$ & $-0,293$ & $-0,700^{\star *}$ & $0,752^{\star * \star}$ & $-0,079$ \\
\hline Grasa intermuscular & $-0,439^{\star}$ & 0,389 & $-0,016$ & $-0,308$ & 0,433 & $-0,095$ \\
\hline Grasa subcutánea & $-0,252$ & 0,326 & $-0,275$ & $-0,479^{*}$ & 0,419 & 0,003 \\
\hline Hueso & $0,479^{*}$ & $-0,412$ & $0,579^{* *}$ & $-0,126$ & 0,010 & $0,575^{\star *}$ \\
\hline \multicolumn{7}{|l|}{ Pierna } \\
\hline Músculo & $0,741^{\star * *}$ & $-0,608^{* *}$ & 0,140 & $0,634^{\star *}$ & $-0,563^{\star *}$ & 0,143 \\
\hline Grasa total & $-0,798^{* * *}$ & $0,836^{* * *}$ & $-0,377$ & $-0,628^{* *}$ & $0,723^{\star * *}$ & $-0,285$ \\
\hline Grasa intermuscular & $-0,327$ & $0,446^{*}$ & $-0,023$ & 0,000 & 0,068 & $-0,022$ \\
\hline Grasa subcutánea & $-0,710^{\star * *}$ & $0,629^{* *}$ & $-0,480^{\star}$ & $-0,622^{\star *}$ & $0,657^{\star *}$ & $-0,263$ \\
\hline Hueso & 0,381 & $-0,422$ & $0,871^{\star \star *}$ & 0,004 & $-0,259$ & $0,546^{*}$ \\
\hline \multicolumn{7}{|l|}{ Bajos } \\
\hline Músculo & $0,858^{* * *}$ & $-0,805^{\star * *}$ & $0,554^{* *}$ & $0,566^{\star *}$ & $-0,566^{\star *}$ & $-0,172$ \\
\hline Grasa total & $-0,814^{* * *}$ & $0,892^{* * *}$ & $-0,531$ & $-0,612^{* \star}$ & $0,684^{* * *}$ & $-0,034$ \\
\hline Grasa intermuscular & $-0,506^{\star}$ & 0,614 & $-0,216$ & $-0,469^{*}$ & $0,571^{\star *}$ & 0,014 \\
\hline Grasa subcutánea & $-0,439^{\star}$ & 0,417 & $-0,411$ & 0,118 & $-0,211$ & $-0,063$ \\
\hline Hueso & 0,269 & $-0,321$ & $0,656^{\star * *}$ & 0,040 & $-0,060$ & $0,578^{* *}$ \\
\hline \multicolumn{7}{|l|}{ Badal } \\
\hline Músculo & $0,593^{\star \star}$ & $-0,437^{*}$ & 0,382 & $0,576^{\star *}$ & $-0,554^{*}$ & 0,256 \\
\hline Grasa total & $-0,560^{\star *}$ & $0,624^{\star *}$ & $-0,300$ & $-0,450^{*}$ & $0,534^{*}$ & $-0,308$ \\
\hline Grasa intermuscular & $-0,350$ & $0,433^{* *}$ & $-0,207$ & $-0,490^{*}$ & $0,594^{* *}$ & $-0,171$ \\
\hline Grasa subcutánea & $-0,341$ & 0,342 & $-0,165$ & 0,160 & $-0,210$ & 0,125 \\
\hline Hueso & 0,406 & $-0,388$ & $0,570^{\star *}$ & $-0,337$ & 0,278 & 0,267 \\
\hline \multicolumn{7}{|l|}{ Cuello } \\
\hline Músculo & $0,559^{\star \star}$ & $-0,559^{* *}$ & 0,100 & $0,576^{* \star}$ & $-0,554^{*}$ & 0,256 \\
\hline Grasa total & $-0,382$ & 0,420 & $-0,252$ & $0,458^{*}$ & $-0,375$ & 0,105 \\
\hline Grasa intermuscular & $-0,198$ & 0,222 & $-0,128$ & $-0,392$ & 0,381 & $-0,511^{*}$ \\
\hline Grasa subcutánea & $-0,122$ & 0,129 & $-0,084$ & $-0,376$ & 0,431 & $-0,407$ \\
\hline Hueso & 0,038 & 0,022 & $0,691^{* * *}$ & $-0,107$ & 0,008 & $-0,258$ \\
\hline
\end{tabular}

Archivos de zootecnia vol. 60, núm. 232, p. 1128. 


\section{PREDICCIÓN DE LA COMPOSICIÓN TISULAR DE LA CANAL EN OVINOS CANARIOS}

peso de sacrificio. Martínez-Cerezo et al. (2002) comparando corderos de $10-12 \mathrm{~kg} v \mathrm{~s}$ 20-22 kg observó que el porcentaje de músculo aumentaba en la raza Rasa Aragonesa y Churra, en cambio en Merino español no existieron diferencias significativas entre pesos. Por el contrario, Pérez et al. (2002) encontró que el porcentaje de músculo des-

Tabla III. Correlaciones entre los componentes titulares de las diferentes piezas y los de la media canal en la raza Canaria de Pelo. (Correlations among tissue composition of different joints and tissue composition of carcass in Canarian Haired breed).

\begin{tabular}{|c|c|c|c|c|c|c|}
\hline \multirow{2}{*}{$\begin{array}{l}\text { Pieza } \\
\text { Tejidos }\end{array}$} & \multicolumn{3}{|c|}{$15 \mathrm{~kg}$} & \multicolumn{3}{|c|}{$24 \mathrm{~kg}$} \\
\hline & Músculo & Grasa total & Hueso & Músculo & Grasa total & Hueso \\
\hline \multicolumn{7}{|l|}{ Costillar } \\
\hline Músculo & $0,875^{* * *}$ & $-0,837^{* * *}$ & 0,075 & $0,740^{* * *}$ & $-0,782^{* * *}$ & $-0,144$ \\
\hline Grasa total & $-0,673^{* * *}$ & $0,897^{\star * *}$ & $-0,263$ & $-0,591^{* *}$ & $0,793^{\star * *}$ & $-0,135$ \\
\hline Grasa intermuscular & $-0,364$ & $0,663^{* * *}$ & $-0,390$ & $-0,065$ & 0,390 & $-0,463^{*}$ \\
\hline Grasa subcutánea & $-0,665^{\star * *}$ & $0,647^{* *}$ & 0,074 & $-0,636^{* *}$ & $0,508^{*}$ & 0,365 \\
\hline Hueso & 0,277 & 0,113 & 0,370 & $-0,358$ & 0,229 & 0,419 \\
\hline \multicolumn{7}{|l|}{ Espalda } \\
\hline Músculo & $0,694^{* * *}$ & $-0,367$ & $-0,272$ & $0,655^{\star * *}$ & $-0,552^{*}$ & $-0,257$ \\
\hline Grasa total & $-0,509^{\star}$ & $0,481^{*}$ & $-0,004$ & $-0,518^{*}$ & $0,469^{*}$ & 0,451 \\
\hline Grasa intermuscular & $-0,135$ & 0,217 & 0,107 & $-0,174$ & 0,108 & 0,284 \\
\hline Grasa subcutánea & $-0,522^{*}$ & 0,439 & $-0,069$ & $-0,554^{\star}$ & $0,551^{*}$ & 0,351 \\
\hline Hueso & $-0,391$ & 0,030 & $0,632^{\star *}$ & $-0,316$ & 0,122 & 0,160 \\
\hline \multicolumn{7}{|l|}{ Pierna } \\
\hline Músculo & $0,815^{\star * *}$ & $-0,642^{* *}$ & $-0,237$ & $0,672^{\star * *}$ & $-0,406$ & $0,672^{* * *}$ \\
\hline Grasa total & $-0,585^{\star *}$ & $0,865^{\star * *}$ & $-0,501^{*}$ & $-0,583^{* *}$ & $0,780^{\star \star \star}$ & $-0,583^{* *}$ \\
\hline Grasa intermuscular & 0,053 & 0,314 & $-0,589^{\star \star}$ & $-0,183$ & $0,582^{* \star}$ & $-0,183$ \\
\hline Grasa subcutánea & $-0,699^{\star * *}$ & $0,769^{\star * *}$ & $-0,171$ & $-0,447$ & 0,220 & $-0,447$ \\
\hline Hueso & $-0,162$ & $-0,066$ & $0,728^{\star \star \star}$ & $-0,060$ & $-0,342$ & $-0,060$ \\
\hline \multicolumn{7}{|l|}{ Bajos } \\
\hline Músculo & $0,692^{* * *}$ & $-0,535^{\star}$ & $0,692^{\star * \star}$ & $0,628^{\star *}$ & $-0,551^{\star}$ & 0,084 \\
\hline Grasa total & $-0,633^{\star *}$ & $0,723^{\star * *}$ & $-0,633^{\star \star}$ & $-0,497^{\star}$ & $0,737^{\star \star \star}$ & $-0,329$ \\
\hline Grasa intermuscular & $-0,116$ & 0,347 & $-0,116$ & $-0,104$ & 0,340 & $-0,401$ \\
\hline Grasa subcutánea & $-0,565^{\star *}$ & $0,472^{*}$ & $-0,565^{\star \star}$ & $-0,645^{\star *}$ & $0,660^{\star *}$ & 0,103 \\
\hline Hueso & 0,517 & $-0,516^{*}$ & 0,517 & $-0,051$ & $-0,120$ & 0,392 \\
\hline \multicolumn{7}{|l|}{ Badal } \\
\hline Músculo & $0,634^{* *}$ & $-0,420$ & $-0,136$ & $0,544^{*}$ & $-0,209$ & $-0,420$ \\
\hline Grasa total & $-0,403$ & 0,430 & 0,068 & $-0,208$ & 0,055 & $-0,076$ \\
\hline Grasa intermuscular & $-0,387$ & 0,425 & $-0,059$ & 0,156 & $-0,061$ & $-0,376$ \\
\hline Grasa subcutánea & $-0,093$ & 0,078 & 0,230 & $-0,469^{*}$ & 0,148 & 0,360 \\
\hline Hueso & $-0,248$ & 0,115 & 0,147 & $-0,134$ & $-0,101$ & $0,626^{* *}$ \\
\hline \multicolumn{7}{|l|}{ Cuello } \\
\hline Músculo & $0,564^{\star *}$ & $-0,362$ & 0,030 & $0,628^{* *}$ & $-0,389$ & $-0,187$ \\
\hline Grasa total & $-0,638^{\star *}$ & $0,744^{* *}$ & $-0,270$ & $-0,762^{\star * *}$ & $0,631^{* *}$ & 0,183 \\
\hline Grasa intermuscular & $-0,500^{*}$ & $0,711^{* * *}$ & $-0,322$ & $-0,605^{\star *}$ & $0,606^{\star *}$ & 0,091 \\
\hline Grasa subcutánea & $-0,278$ & 0,162 & 0,024 & -0.217 & $-0,038$ & 0,164 \\
\hline Hueso & 0,031 & $-0,289$ & 0,364 & $0,531^{*}$ & $-0,448$ & 0,050 \\
\hline
\end{tabular}


cendía con el crecimiento. Míguelez et al. (2007) señalan que la proporción de hueso disminuye cuando aumenta el peso, debido a que es el tejido más precoz. En las figuras 1 y $\mathbf{2}$ observamos que la canal de la raza de pelo presenta más porcentaje de músculo y menor porcentaje de grasa que la raza de lana. Algunos autores (Sañudo et al., 1997; Vergara et al., 1997) han relacionado la aptitud productiva de la raza con el contenido graso de la canal, señalando que las razas lecheras acumularían más grasa que las de carne, en canales pesadas.

Obtenidas las correlaciones entre los

Tabla IV. Valores de $R^{2}$ corregida de las ecuaciones predictoras obtenidas, según la pieza diseccionada, para las dos razas. (Adjusted $\mathrm{R}^{2}$ for the prediction equations for the different joints in both breeds).

\begin{tabular}{|c|c|c|c|c|}
\hline \multirow{2}{*}{$\begin{array}{l}\text { Pieza } \\
\quad \text { Tejidos (\%) }\end{array}$} & \multicolumn{2}{|c|}{ Canaria } & \multicolumn{2}{|c|}{ Canaria de Pelo } \\
\hline & $15 \mathrm{~kg}$ & $24 \mathrm{~kg}$ & $15 \mathrm{~kg}$ & $24 \mathrm{~kg}$ \\
\hline \multicolumn{5}{|l|}{ Espalda } \\
\hline Músculo & $0,493^{* *}$ & $0,419^{\star \star *}$ & $0,453^{\star \star *}$ & $0,395^{\star *}$ \\
\hline Grasa total & $0,197^{*}$ & $0,424^{* * *}$ & - & $0,264^{*}$ \\
\hline Hueso & $0,300^{* *}$ & $0,293^{* *}$ & $0,366^{* *}$ & - \\
\hline \multicolumn{5}{|l|}{ Cuello } \\
\hline Músculo & $0,276^{\star *}$ & $0,166^{\star}$ & $0,280^{* *} C$ & $0,671^{* * *}$ \\
\hline Grasa total & $0,276^{* *}$ & - & $0,566^{* * *}$ & $0,329^{\text {** }}$ \\
\hline Hueso & $0,450^{\star \star *}$ & $0,350^{* *}$ & 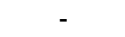 & \\
\hline \multicolumn{5}{|l|}{ Pierna } \\
\hline Músculo & $0,653^{* * *}$ & $0,527^{* *}$ & $0,645^{* * *}$ & $0,419^{* *}$ \\
\hline Grasa total & $0,697^{* \star *}$ & $0,545^{\star * *}$ & $0,734^{* * *} C$ & $0,582^{* * *}$ \\
\hline Hueso & $0,746^{\star * *}$ & $0,259^{*}$ & $0,588^{* * *} C$ & $0,428^{* * *}$ \\
\hline \multicolumn{5}{|l|}{ Bajos } \\
\hline Músculo & $0,722^{\star * \star}$ & $0,307^{*}$ & $0,636^{* * *} \mathrm{C}$ & $0,646^{* * *}$ \\
\hline Grasa total & $0,800^{\star * *}$ & $0,406^{\star *}$ & $0,438^{\star *}$ & $0,698^{\star * *}$ \\
\hline Hueso & $0,575^{\star * *}$ & $0,297^{* *}$ & - & \\
\hline \multicolumn{5}{|l|}{ Costillar } \\
\hline Músculo & $0,813^{* * *}$ & $0,919^{* * *}$ & $0,753^{\star * \star} c$ & $0,521^{* * *}$ \\
\hline Grasa total I & $0,854^{* * *}$ & $0,873^{* * *}$ & $0,766^{\star * \star}$ & $0,588^{\star * *}$ \\
\hline Hueso & $0,380^{* *}$ & $0,275^{*}$ & - & $0,169^{*}$ \\
\hline \multicolumn{5}{|l|}{ Badal } \\
\hline Músculo & $0,444^{* *}$ & $0,482^{* *}$ & $0,652^{\star * *}$ & $0,254^{\star}$ \\
\hline Grasa total & $0,318^{* *}$ & $0,561^{* * *}$ & - & \\
\hline Hueso & $0,289^{\star *}$ & - & - & $0,357^{\star *}$ \\
\hline
\end{tabular}

tejidos de la media canal y los de sus diferentes piezas (tablas II y III), en los cuatro tipos de canales el costillar fue la pieza que presentó las correlaciones más altas para el porcentaje de músculo y grasa, y la pierna para el porcentaje de hueso. Díaz (2001) también encontró que el costillar era la pieza que presentaba mejores correlaciones, siendo altas para los tres tejidos. Luaces et al. (2008) en corderos de $15 \mathrm{~kg}$ observó que la espalda era la pieza más correlacionada con los diferentes tejidos de la canal, no obstante, en los corderos de $21 \mathrm{~kg}$ de raza Gallega fueron la pierna y la espalda las piezas mejor correlacionadas con los tejidos de la canal.

En la tabla IV se exponen los valores de $\mathrm{R}^{2}$ corregida correspondientes a las ecuaciones de predicción obtenidas del análisis de regresión múltiple. En los corderos de lana el costillar fue la pieza que presentó los coeficientes de regresión más elevados para el músculo y la grasa. En los corderos de pelo el costillar fue también la pieza con mejores coeficientes para el músculo y grasa, pero sólo en los corderos más ligeros, pues en los corderos pesados fueron los bajos los que presentaron los mejores resultados. En ambas razas, el hueso fue un tejido que presentó baja predicción, algo que no hemos encontrado que suceda con otras razas ovinas. En los cuatro tipos de canales, la pierna fue la pieza que ofreció los coeficientes más elevados para el hueso. Ruiz de Huidobro y Cañeque, (1994); Miguélez et al. (2006) encontraron que el costillar y la pierna fueron los mejores predictores para el músculo, grasa y hueso en corderos Manchegos y de de la IGP Lechazo de Castilla y León, respectivamente. Las tablas V y VI recogen las ecuaciones de predicción de la composición tisular de la canal para cada tipo de cordero. Debido a la baja predicción del hueso, se incorporó a la regresión múltiple, como variable independiente, el peso de la media canal, para comprobar si esto mejoraba el valor de $\mathrm{R}^{2}$ corregida. Sólo en la raza Canaria se logra mejorar la predicción al añadir el peso de la 

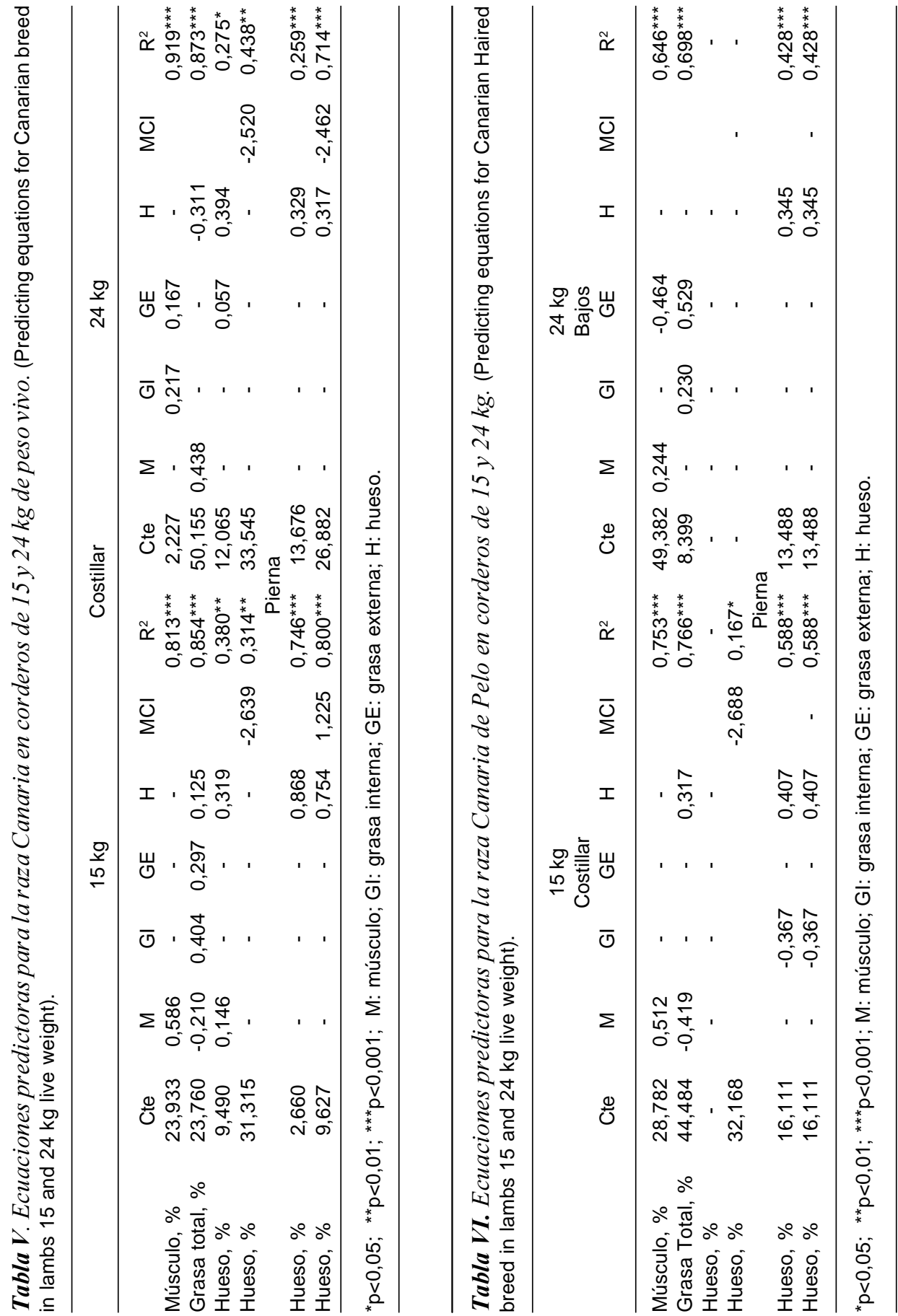


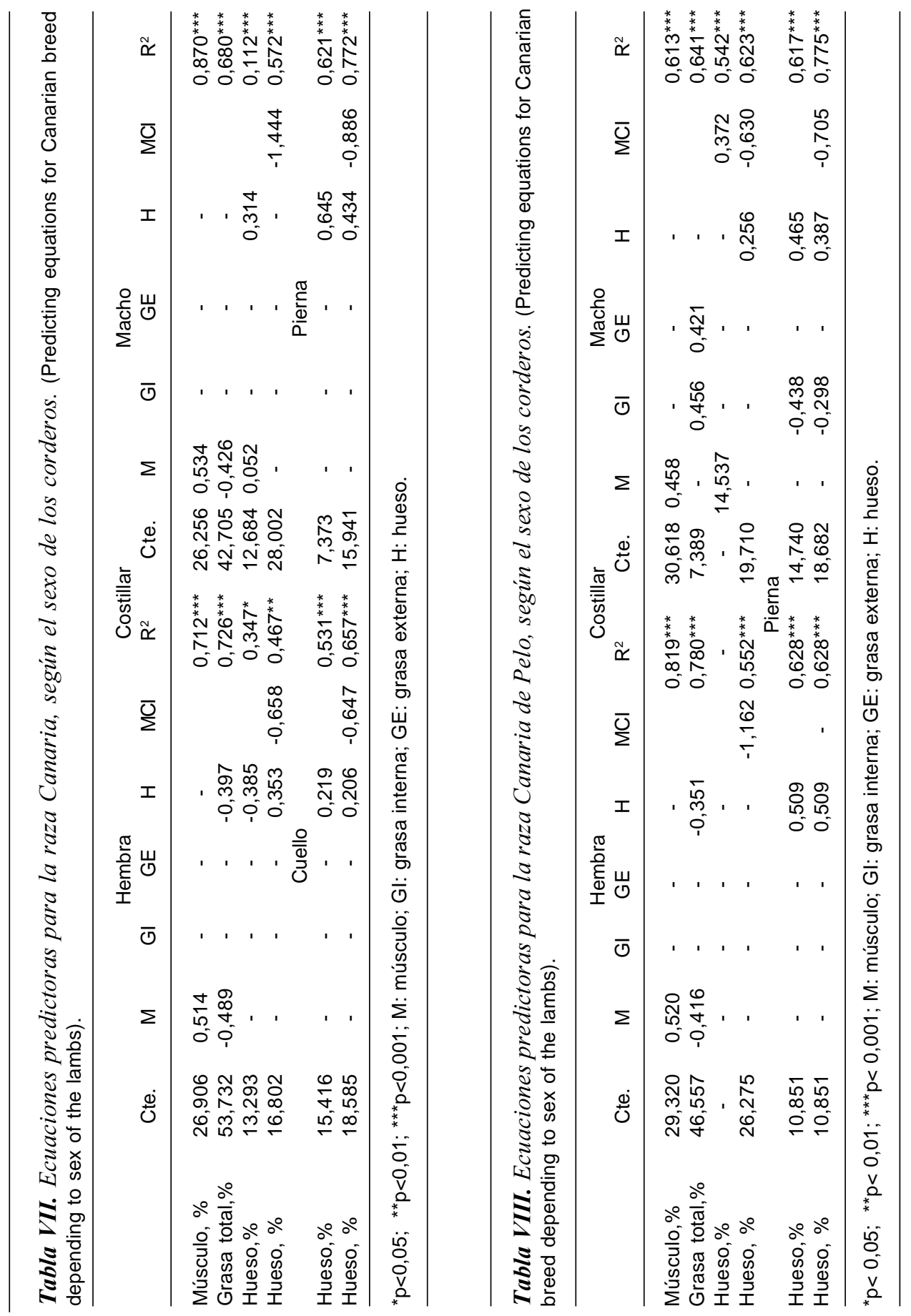


media canal, no ocurriendo lo mismo con la raza Canaria de Pelo.

En las tablas VII y VIII se recogen las ecuaciones de predicción para cada raza en función del sexo de los corderos. En la raza Canaria no se produce una mejora de la predicción de la composición tisular de la cana al separar las canales en función del sexo. No obstante, en la raza Canaria de Pelo, se obtuvieron coeficientes de regresión más elevados para todos los tejidos, por lo que para esta raza estas ecuaciones resultan más precisas. En este modelo de ecuaciones por sexos también se valoró si el peso de la media canal mejoraba la predicción del hueso, comprobando que así era en todos los casos, pues aumentó el coeficiente de regresión de las ecuaciones.

La utilización de una única ecuación por raza, sin tener en cuenta el peso ni el sexo de los corderos, también ha sido considerada, y se recoge en la tabla IV. Aunque la raza Canaria presentó mejor predicción de los tejidos de la canal que la raza Canaria de Pelo, los coeficientes de regresión no son mejores que los obtenidos por peso y sexo, para ambas razas. El tejido que presentó mejor predicción fue el músculo, seguido de la grasa y el hueso. Para Díaz et al., 2004 y Ruiz de Huidobro y Cañeque, 1994 la grasa ha sido el tejido que se ha predicho con mayor exactitud.

Según los resultados obtenidos, la mejor predicción de los tejidos de la canal se consigue utilizando ecuaciones en función del pesos vivo de los corderos en de la raza Canaria, y ecuaciones en función del sexo de los corderos en la raza Canaria de Pelo.

\section{CONCLUSIONES}

La raza Canaria fue la que presentó mejor predicción, especialmente para el porcentaje de músculo y grasa. En cualquier caso, las ecuaciones deben emplearse en corderos de la misma raza y peso, y criados en condiciones similares a las de

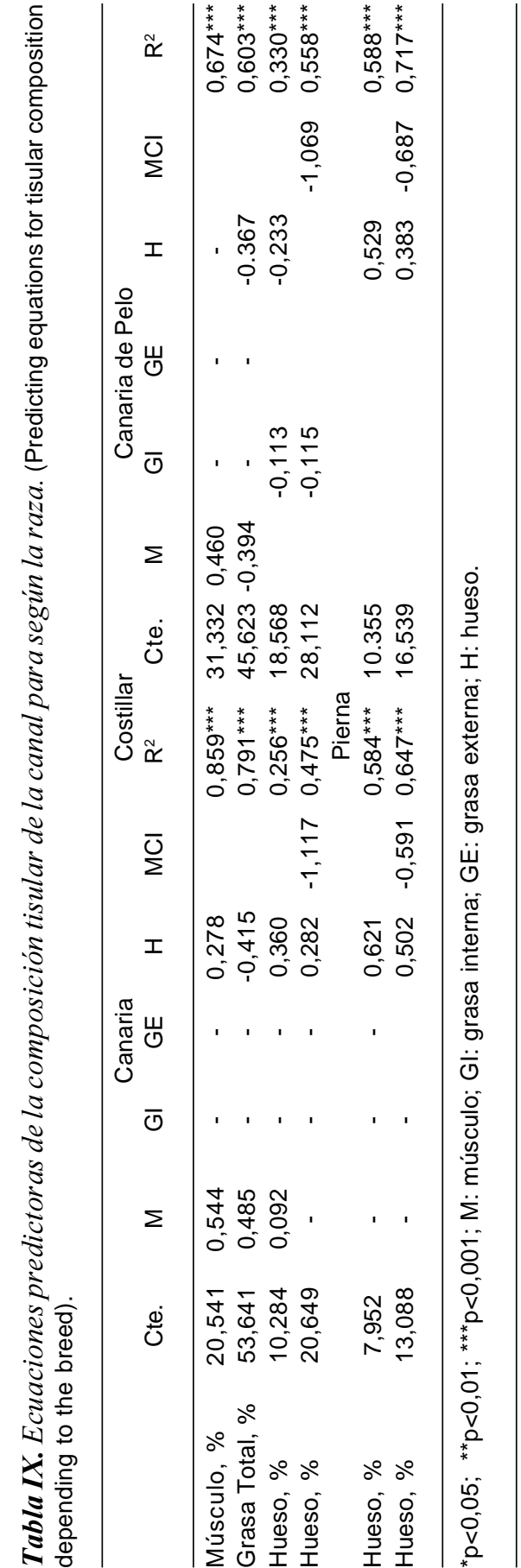




\section{CAMACHO, PÉREZ, MATA YBERMEJO}

este trabajo. Aunque la predicción de la composición tisular de la canal, a través de datos de disección de algunas piezas, ha resultado un método preciso para diferentes autores, en nuestro caso resulta necesario ampliar el muestreo, en ambas razas, para mejorar los coeficientes de determinación para los diferentes tejidos, y disponer así

\section{BIBLIOGRAFÍA}

Blázquez, B., Onega, E., Miguel, E. y Ruiz de Huidobro, F. 2002. Evolución comparativa de las piezas de carnicería y de las normalizadas en el cordero, en función del aumento del peso vivo. XXVII Jornadas Científicas y VI Jornadas Internacionales de la SEOC. Valencia. España. pp: 270-274.

Boccard, R., Dumont, B.I. et Lefebvre, J. 1976. Étude de la production de la viande chez les ovins. X. Relations entre la composition anatomique des différentes régions corporelles de I'agneau. Ann. Zootech. 25: 95-110.

Camacho, A., Bermejo, L.A. y Mata, J. 2007. Análisis del potencial del ovino de pelo. Arch. Zootec., 56: 507-510.

Colomer-Rocher, F., Delfa, R. y Sierra, I. 1988. Método normalizado para el estudio de los caracteres cuantitativos y cualitativos de las canales ovinas producidas en el área mediterránea, según los sistemas de producción. Cuadernos INIA, 17: 19-41.

Delgado, J.V., Fresno, M., Camacho, M.E., Rodero, E. y Barba C. 1998. Origen e influencias del ovino canario. Arch. Zootec., 47: 511-516.

Díaz, M.T. 2001. Características de la canal de corderos lechales manchegos. Correlaciones y ecuaciones de predicción. Tesis doctoral. Universidad Complutense. Madrid.

Díaz, M.T., Cañeque, V., Lauzurica, S., Velasco, S., Ruíz de Huidobro, F. and Pérez, C. 2004. Prediction of suckling lambs carcass composition from objective and subjective carcass measurements. Meat Sci., 66: 895-902.

Diestre, A. 1985. Estudio de los factores biológicos determinantes del desarrollo de las canales de cordero y de sus características comerciales. Tesis doctoral. Universidad de Zaragoza. I.S.T.A.C. 2006. Estadística Agraria de Canarias. de ecuaciones más precisas.

\section{AGRADECIMIENTOS}

Al INIA por la financiación del proyecto de investigación (RTA/2006-00174-00-00), a la granja Gorón Sur S.L y al matadero insular de Tenerife.

Consejería de Agricultura, Ganadería y Pesca. Islas Canarias.

Kempster, A.J. 1981. The indirect evaluation of sheep carcass composition in breeding schemes, population studies and experiments. Livest. Prod. Sci., 8: 263-271.

López, M. 1987. Calidad de la canal y de la carne en los tipos de lechal, ternasco y cordero de la raza Lacha y estudio de su desarrollo. Tesis Doctoral. Universidad de Zaragoza.

Luaces, M., Calvo, C., Fernández, A., Viana, J.L. y Sánchez, L. 2008. Ecuaciones predictoras de la composición tisular de las canales de corderos de raza Gallega. Arch. Zootec., 57: 1-12.

Martínez-Cerezo, S., Olleta, J.L., Sañudo, C., Delfa, R., Cuartielles, I., Pardos, J.J., Medel, I., Panea, B. y Sierra, I. 2002. Calidad de la canal en tres razas ovinas españolas. Efecto del peso de sacrificio. XXVII Jornadas Científicas y VI Jornadas Internacionales de la SEOC. Valencia España. pp. 288-295.

Miguélez, E., Zumalacárregui, J.M., Osorio, M.T., Beteta, O. and Mateo, J. 2006. Carcass characteristics of sucking lambs protected by the PGI Lechazo de Castilla y León. European quality label: Effect of breed, sex and carcass weight. Meat Sci., 73: 82-89.

Miguélez, E., Zumalacárregui, J.M., Osorio, M.T. y Mateo, J. 2007. Características de la canal de cordero lechal de diversas razas producidas en España. (Revisión bibliográfica). ITEA, 103: 14-30.

Pérez, O. 1992. Instalación de ovino Pelibuey ligada a la agricultura extensiva. Trabajo Fin de Carrera. EUITA. Universidad de La Laguna. Tenerife.

Pérez, P., Maino, M., Tomic, G., Mardones, E. and Pokniak, J. 2002. Carcass characteristics and meat quality of Suffolk Down sucking lambs. 


\section{PREDICCIÓN DE LA COMPOSICIÓN TISULAR DE LA CANAL EN OVINOS CANARIOS}

Small Rum. Res., 44: 233-240.

Ruiz de Huidobro, F. y Cañeque, V. 1994. Producción de carne de corderos de la raza Manchega. IV. Ecuaciones predictoras de la composición tisular de las canales. Invest. Agr. Prod. San. Anim., 9: 71-82.

Sañudo, C. 1980. Calidad de la canal y de la carne en el Ternasco típico Aragonés. Tesis Doctoral. Universidad de Zaragoza. 337 pp.

Sañudo, C., Sierra, I., Olleta, J.L., Conesa, A. y Alcalde, M.J. 1989. La calidad de la canal y de la carne en corderos ligeros tipo ternasco: Competencia con canales de procedencia extranjera.
Actas de las XIV Jornadas Científicas de la S.E.O.C. Jaén. pp. 223-232.

Sañudo, C., Campo, M.M., Sierra, I.M., María, G.A., Olleta, J.L. and Santolaria, P. 1997. Breed effect on carcase and meat quality of suckling lambs. Meat Sci., 46: 357-365.

SPSS. 2006. SPSS 15.0 for Windows. SPSS. Inc. Chicago, USA.

Vergara, H., Fernández, C., García, A., Gallego, L. 1997. Estudio comparativo de la calidad de la canal de corderos de la raza Manchega en función del peso de sacrificio. ITEA, (Vol. Extra): 712-714. 